\title{
Way of Detection of Refusals at Economic Reservation of the Onboard Equipment of a Unmanned Aerial Vehicle
}

\author{
Michael F. Volobuyev, Alexander M. Maltsev*, \\ Sergey B. Mihajlenko and Vladimir A. Ufaev \\ Military Educational and Scientific Centre \\ of the Air Force N.E. Zhukovsky and Y.A Gagarin Air Force Academy \\ 54a Starykh Bolshevikov, Voronezh, 394064, Russia
}

Received 29.07.2016, received in revised form 15.09.2016, accepted 30.10.2016

A method of detection the occurrence of progressive failure in dual-redundant system is proposed. The design procedure of likelihood characteristics of a way is developed and the estimation of its efficiency on a concrete example is spent. Recommendations for choice parameters of a way which minimize acceptance of erroneous decisions on system refusal are offered.

Keywords: dual-redundant system, gradual refusal, a threshold of detection of refusal.

Citation: Volobuyev M.F., Maltsev A.M., Mihajlenko S.B., Ufaev V.A. Way of detection of refusals at economic reservation of the onboard equipment of a unmanned aerial vehicle, J. Sib. Fed. Univ. Eng. technol., 2016, 9(7), 1059-1067. DOI: 10.17516/1999-494X-2016-9-7-1059-1067.

(C) Siberian Federal University. All rights reserved

* Corresponding author E-mail address: max alex_67@mail.ru 


\title{
Способ обнаружения отказов
}

\section{при экономичном резервировании}

\section{бортового оборудования}

\section{беспилотного летательного аппарата}

\author{
М.Ф. Волобуев, А.М. Мальцев, \\ С.Б. Михайленко, В.А. Уфаев \\ Военный учебно-научный цеентр Военно-воздушных сил \\ «Военно-воздушная академия \\ имени профессора Н. Е. Жуковского и Ю. А. Гагарина» \\ Россия, 394064, Воронеж, ул. Старых Большевиков, 54 а
}

Предложен способ обнаружения факта наступления постепенного отказа в двухканальной резервированной системе. Разработана методика расчета вероятностных характеристик способа и проведена оценка его эффективности на конкретном примере. Предложень рекомендации по выбору параметров способа, которые минимизируют принятие ошибочных решений об отказе системы.

Ключевые слова: двухканальная резервированная система, постепенный отказ, порог обнаружения отказа.

\section{Введение}

Резервирование наиболее ответственных, жизненно важных блоков и узлов является эффективным способом повышения безотказности любой технической системы, что объясняет его широкое применение в авиационной технике. С другой стороны, жесткие массогабаритные ограничения, налагаемые на бортовое оборудование летательных аппаратов, не всегда позволяют наращивать число резервных каналов для достижения требуемого уровня безотказности. Наиболее жесткие массогабаритные, а также стоимостные ограничения характерны для беспилотных летательных аппаратов. Поэтому для них весьма актуальна задача разработки схем экономичного резервирования бортового оборудования, при котором число дублирующих каналов минимально [1]. Предельный случай экономичного резервирования двухканальная схема резервирования, при которой резервированная система содержит один дублирующий канал. При реализации таких систем в общем случае невозможно определить место отказа по результатам сравнительного анализа сигналов в двух каналах. Однако при определенных условиях достаточно установить сам факт наступления отказа без определения его локализации. Например, факт наступления отказа может быть использован для подключения к двухканальной резервированной системе дополнительного, более грубого третьего канала, математической модели канала или других устройств, позволяющих определить отказавший канал. Для реализации процедуры обнаружения факта отказа в двухканальной резервированной системе должен быть определен пороговый уровень обнаружения отказа с учетом характера его влияния на вероятности принятия правильных и ошибочных решений о состоянии системы. 
Наиболее просто реализуем способ обнаружения мгновенных отказов, основанный на контроле по пороговой величине. В [2-5] описаны устройства обнаружения отказов, управления и восстановления работоспособности резервированных систем с использованием контроля по пороговой величине. Данные устройства позволяют непрерывно функционировать двухканальной системе при следующих отказах в одном из каналов: скачки выходных параметров вверх (типа короткого замыкания) или вниз (типа обрыв соединения), а также плавное изменение до максимального или минимального значения, возможного в данной системе. Принципиальным недостатком способа контроля по пороговой величине выступает невозможность обнаружения постепенных отказов.

Цель статьи - разработка способа обнаружения факта наступления постепенного отказа в двухканальной резервированной системе и определение его вероятностных характеристик.

\section{Способ обнаружения факта наступления постепенного отказа}

Рассмотрим двухканальную резервированную систему, в которой сигналы с выходов каналов являются случайными некоррелированными процессами. Будем считать, что случайные величины $X$ и $Y$, наблюдаемые на выходе каналов в текущий момент времени, характеризуются одинаковыми вероятностными распределениями с параметрами: $M[X]=M[Y]=m$; $M\left[(X-m)^{2}\right]=M\left[(Y-m)^{2}\right]=\sigma^{2}$. Для сигналов $X$ и $Y$ определим диапазон всех физически возможных значений $\beta$ и диапазон рабочих (номинальных) значений $\alpha$. Диапазон $\alpha$ располагается внутри диапазона $\beta(\alpha<\beta)$ и разбивает его на три поддиапазона: $A_{1}$ - соответствует рабочему диапазону $\alpha$; $A_{2}$ - расположен ниже диапазона $\alpha$; $A_{3}$ - расположен выше диапазона $\alpha$.

Процедура обнаружения факта наступления отказа в двухканальной системе может быть реализована с помощью схемы, представленной на рис. 1.

Обнаружение факта наступления отказа осуществляется в соответствии с критерием

$$
\begin{aligned}
& \Delta>\Delta^{*}-\text { отказ в системе, } \\
& \Delta \leq \Delta^{*}-\text { система исправна, }
\end{aligned}
$$

где $\Delta=|X-Y|, \Delta^{*}-$ пороговый уровень обнаружения отказа, $\Delta \leq \alpha^{*}$.

\section{Методика расчета вероятностных характеристик}

\section{способа обнаружения факта наступления постепенного отказа}

Принятие решения об отказе системы в соответствии с критерием (1) носит вероятностный характер. При этом возможны следующие варианты (исходы), составляющие полную группу несовместных событий:

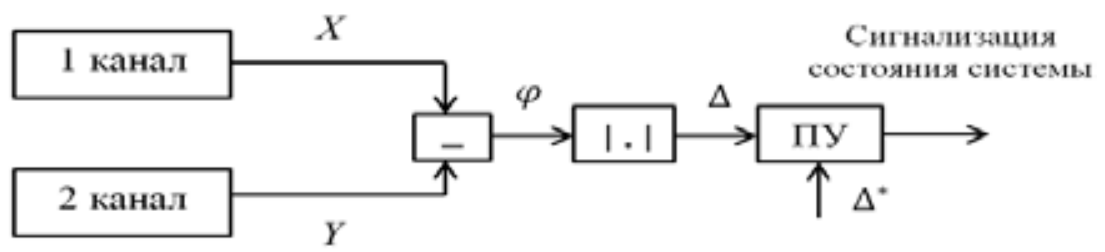

Рис. 1. Схема обнаружения факта наступления отказа в двухканальной резервированной системе 
1) выходные сигналы $X$ и $Y$ находятся в диапазоне значений $A_{1}$ (отказа нет), а модуль их разности $\Delta$ не превысил порог $\Delta^{*}$ (правильное решение об исправности системы);

2) выходные сигналы $X$ и $Y$ находятся в диапазоне значений $A_{1}$ (отказа нет), а модуль их разности $\Delta$ превысил порог (ложное решение об отказе системы);

3) один или оба сигнала $X, Y$ находятся в диапазоне значений $A_{2}$ или $A_{3}$ (отказ одного или обоих каналов), а модуль разности сигналов $\Delta$ превысил порог (правильное решение об отказе системы);

4) один или оба сигнала $X, Y$ находятся в диапазоне значений $A_{2}$ или $A_{3}$ (отказ одного или обоих каналов), а модуль разности сигналов $\Delta$ не превысил порог (ложное решение об исправности системы).

Граф состояний системы с возможными исходами процедуры обнаружения отказа представлен на рис. 2, где приняты следующие обозначения: $\mathrm{HC}$ - начальное состояние системы; Отк - отказ системы; Исп - исправность системы; ПРО - правильное решение об отказе системы; ЛРИ - ложное решение об исправности системы; ПРИ - правильное решение об исправности системы; ЛРО - ложное решение об отказе системы; $P_{\text {отк }}$ вероятность отказа системы; $P_{\text {исп }}-$ вероятность исправности системы; $P_{\text {ппотк }}-$ вероятность превышения порога в случае отказа системы; $P_{\text {нпотк }}-$ вероятность непревышения порога в случае отказа системы; $P_{\text {пписп }}-$ вероятность превышения порога в случае исправности системы; $P_{\text {нписп }}-$ вероятность непревышения порога в случае исправности системы; $P_{\text {прос }}$ вероятность правильного решения об отказе системы; $P_{\text {лрис }}-$ вероятность ложного решения об исправности системы; $P_{\text {прис }}-$ вероятность правильного решения об исправности системы; $P_{\text {лрос }}-$ вероятность ложного решения об отказе системы.

Введем описание исправного $S_{\text {исп }}$ и неисправных состояний системы $S_{\text {отк }}(i, j)$ :

$$
\begin{aligned}
& S_{\text {исп }}=X \in A_{1} \wedge Y \in A_{1}, \\
& S_{\text {отк }}(i, j)=X \in A_{i} \wedge Y \in A_{j}, i, j=\overline{1,3} .
\end{aligned}
$$

Причем $S_{\text {отк }}(1,1)=\varnothing$, так как этот случай соответствует исправному состоянию $S_{\text {исп }}$.

Вероятности введенных состояний равны:

$$
P_{\text {исп }}=\int_{A_{1}} \omega_{X}(X) \cdot \int_{A_{1}} \omega_{Y}(Y) d Y d X
$$

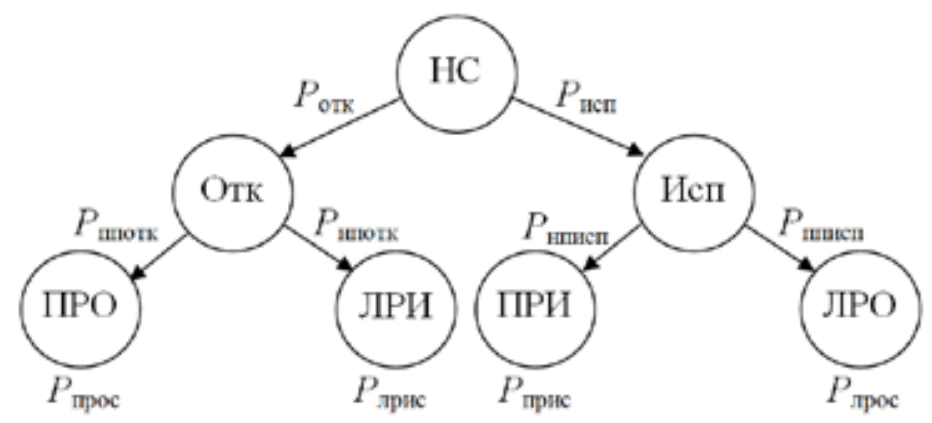

Рис. 2. Граф состояний двухканальной резервированной системы 


$$
P_{\text {отк }}(i, j)=\int_{A_{i}} \omega_{X}(X) \cdot \int_{A_{j}} \omega_{Y}(Y) d Y d X, i, j=\overline{1,3}
$$

где $\omega_{X}(X), \omega_{Y}(Y)$ - плотности вероятности случайных величин $X$ и $Y$.

В случае исправной системы (состояние $S_{\text {ипп }}$ ) возможно правильное решение об её исправности или ложное решение об её отказе, вероятности которых $P_{\text {прис }}, P_{\text {лрос }}$ равны:

$$
P_{\text {прис }}=P_{\text {исп }} \cdot P_{\text {нписп }}, P_{\text {лрос }}=P_{\text {исп }} \cdot P_{\text {пписп }},
$$

где $P_{\text {пписп }}, P_{\text {нписп }}-$ вероятности превышения и непревышения величиной $\Delta$ порога $\Delta^{*}$ в случае исправности системы;

$$
P_{\text {нписп }}=\int_{0}^{\Delta^{*}} \omega_{\Delta}\left(\Delta / A_{1}, A_{1}\right) d \Delta, P_{\text {пписп }}=\int_{\Delta^{*}}^{\alpha} \omega_{\Delta}\left(\Delta / A_{1}, A_{1}\right) d \Delta,
$$

где $\omega_{\Delta}\left(\Delta / A_{1}, A_{1}\right)$ - условная плотность вероятности величины $\Delta$ при попадании $X$ и $Y$ в диапазон $A_{1}$.

В случае неисправной системы (состояния $\mathrm{S}_{\text {отк }}(i, j), i, j=\overline{1,3}$ ) возможны правильные решения об её отказе или ложные решения об её исправности, вероятности которых $P_{\text {прос }}(i, j)$, $P_{\text {лрис }}(i, j)$ равны:

$$
P_{\text {прос }}(i, j)=P_{\text {отк }}(i, j) \cdot P_{\text {пाотк }}(i, j), P_{\text {лрис }}(i, j)=P_{\text {отк }}(i, j) \cdot P_{\text {нпотк }}(i, j), i, j=\overline{1,3}
$$

где $P_{\text {ппок }}(i, j), P_{\text {нпот }}(i, j)$ вероятности превышения и непревышения величиной $\Delta$ порога $\Delta^{*}$ в случае отказа системы;

$$
P_{\text {ппотк }}(i, j)=\int_{\Delta^{*}}^{\Delta_{\text {max }}(i, j)} \omega_{\Delta}\left(\Delta / A_{i}, A_{j}\right) d \Delta, P_{\text {нпотк }}(i, j)=\int_{\Delta_{\text {min }}(i, j)}^{\Delta^{*}} \omega_{\Delta}\left(\Delta / A_{i}, A_{j}\right) d \Delta, i, j=\overline{1,3}
$$

где $\omega_{\Delta}\left(\Delta / A_{i}, A_{j}\right)$ - условная плотность вероятности величины $\Delta$ при попадании $X$ и $Y$ в диапазон $A_{i}$ и $A_{j}$ соответственно, а $\Delta_{\min }(i, j), \Delta_{\max }(i, j)$ - минимальные и максимальные значения величины $\Delta$ в ситуации $S_{\text {отк. }}$ С учетом всех состояний отказа системы получаем вероятности правильного решения об отказе $P_{n р о с}$ и ложного решения об исправности $P_{\text {лрис }}$ :

$$
P_{\text {прос }}=\sum_{i=1}^{3} \sum_{j=1}^{3} P_{\text {прос }}(i, j) ; P_{\text {лрис }}=\sum_{i=1}^{3} \sum_{j=1}^{3} P_{\text {лрис }}(i, j)
$$

Исходы, соответствующие правильным и ложным решениям о состоянии системы, могут быть объединены в благоприятный и неблагоприятный исходы с соответствующими вероятностями правильного $P_{П Р}$ и ложного $P_{Л Р}$ решений:

$$
P_{\text {ПГР }}=P_{\text {прис }}+P_{\text {прос }}, P_{\text {ЛР }}=P_{\text {лрис }}+P_{\text {лрос }} .
$$

Поскольку благоприятные и неблагоприятные исходы являются противоположными событиями, то их вероятности связаны соотношением

$$
P_{\mathrm{\Pi P}}+P_{\mathrm{JP}}=1
$$

Выбор порога $\Delta^{*}$ следует осуществлять, исходя из минимизации $P_{Л P}$ и максимизации $P_{I P}$ : 
$$
\Delta_{\text {opt }}^{*}=\operatorname{Arg} \max _{\Delta^{*} \in[0, \alpha]} P_{\text {ПP }}\left(\Delta^{*}\right)=\underset{\Delta^{*} \in[0, \alpha]}{\operatorname{Arg} \min } P_{\text {JP }}\left(\Delta^{*}\right)
$$ \\ Пример расчета вероятностных характеристик способа обнаружения факта наступления постепенного отказа}

Рассмотрим случай, когда случайные величины $X$ и $Y$ распределены по равномерному закону в диапазоне возможных значений $\beta$, причем зона $\alpha$ расположена симметрично относительно математических ожиданий $X$ и $Y$. При этом значения $\beta$ выбираем, исходя из заданной веро-

ятности безотказной работы каждого из каналов $P_{\kappa}$ в соответствии с выражением $P_{\text {к }}=\frac{\alpha}{\beta}$, что обусловлено равномерным законом распределения выходных величин $X$ и $Y$. Для определения конечных исходов необходимо знать условные плотности вероятностей $\omega_{\Delta}\left(\Delta / A_{i}, A_{j}\right) i, j=\overline{1,3}$, которые могут быть определены по методике, предложенной в [6]. Для расчетов по методике [6] требуются условные плотности вероятностей случайных величин $X$ и $Y$ при условии их нахождения в диапазоне $A_{i}, i=\overline{1,3}$. Очевидно, что это будут равномерные плотности вероятностей и их определение тривиально.

В силу симметрии рассматриваемого случая справедливы следующие соотношения:

$$
\begin{aligned}
& \omega_{\Delta}\left(\Delta / A_{1}, A_{2}\right)=\omega_{\Delta}\left(\Delta / A_{1}, A_{3}\right)=\omega_{\Delta}\left(\Delta / A_{2}, A_{1}\right)=\omega_{\Delta}\left(\Delta / A_{3}, A_{1}\right), \\
& \omega_{\Delta}\left(\Delta / A_{2}, A_{2}\right)=\omega_{\Delta}\left(\Delta / A_{3}, A_{3}\right), \omega_{\Delta}\left(\Delta / A_{2}, A_{3}\right)=\omega_{\Delta}\left(\Delta / A_{3}, A_{2}\right) .
\end{aligned}
$$

Кроме того, нет необходимости определять условные плотности вероятностей $\omega_{\Delta}\left(\Delta / A_{2}, A_{3}\right)$ и $\omega_{\Delta}\left(\Delta / A_{3}, A_{2}\right)$, так как при одновременном попадании случайных величин $X$ и $Y$ в диапазоны $A_{2}$ и $A_{3}\left(A_{3}\right.$ и $\left.A_{2}\right)$ соответственно величина $\Delta$ всегда будет больше порога $\Delta^{*} \leq \alpha$, т. е. справедливо

$$
P_{\text {нпотк }}(2,3)=P_{\text {нпотк }}(3,2)=0, P_{\text {ппотк }}(2,3)=P_{\text {ппотк }}(3,2)=1 \text {. }
$$

Таким образом, достаточно определить три условные плотности вероятностей $\omega_{\Delta}\left(\Delta / A_{i}, A_{j}\right)$ :

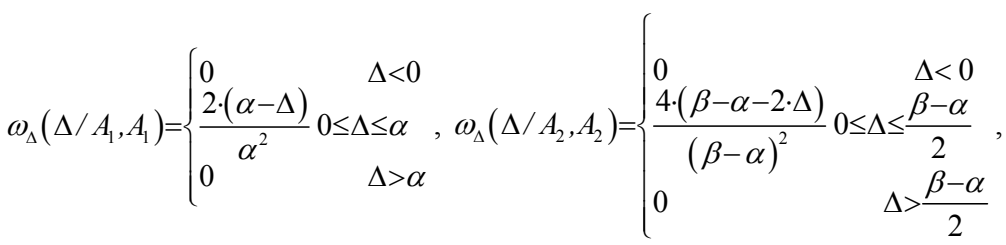

$$
\begin{aligned}
& \omega_{\Delta}\left(\Delta / A_{1}, A_{2}\right)=\left\{\begin{array}{lc}
0 & \Delta<0 \\
\frac{2 \cdot \Delta}{\beta \cdot \alpha-\alpha^{2}} & 0 \leq \Delta<\frac{\alpha}{2} \\
\frac{(\beta-\alpha)}{\beta \cdot \alpha-\alpha^{2}} & \frac{\alpha}{2} \leq \Delta<\alpha \\
\frac{\beta+\alpha-2 \cdot \Delta}{\beta \cdot \alpha-\alpha^{2}} & \alpha \leq \Delta<\frac{\beta+\alpha}{2} \\
0 & \Delta \geq \frac{\beta+\alpha}{2}
\end{array}\right.
\end{aligned}
$$


В результате расчетов с использованием выражений (3)-(8), (12)-(15) определены аналитические выражения для вероятностей конечных исходов:

$$
\begin{aligned}
& P_{\text {прис }}=\left\{\begin{array}{ll}
\frac{2 \cdot \alpha \cdot \Delta^{*}-\Delta^{* 2}}{\beta^{2}} & 0<\Delta^{*}<\alpha \\
\frac{\alpha^{2}}{\beta^{2}} & \Delta^{*}=\alpha
\end{array}, P_{\text {трос }}= \begin{cases}\frac{\alpha^{2}-2 \cdot \alpha \cdot \Delta^{*}+\Delta^{* 2}}{\beta^{2}} & 0<\Delta^{*}<\alpha \\
0 & \Delta^{*}=\alpha\end{cases} \right.
\end{aligned}
$$

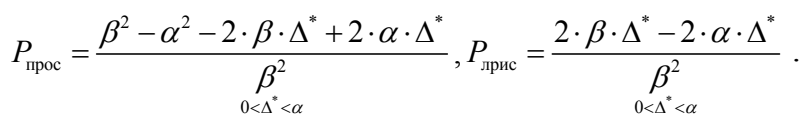

С использованием выражений (16), (17) получены графические зависимости вероятностей ложных и правильных решений, от отношения порога к рабочему диапазону $\Delta^{*} / \alpha$ и фиксированного значения $P_{\mathrm{\kappa}}=\frac{\alpha}{\beta}$, представленные на рис. 3,4 .

Проведя вычисления благоприятных и неблагоприятных решений с использованием выражения (9), получим графические зависимости, представленные на рис. 5.

Из приведенных на рис. 5 зависимостей видно, что существует оптимальное значение порога $\Delta^{*}=\Delta_{\text {opt }}^{*}$, которому соответствует максимальное значение вероятности правильных решений и минимальное значение ложных решений. Причем значения $\Delta_{o p t}^{*}, P_{\text {Пр }}\left(\Delta_{o p t}^{*}\right), P_{\text {пр }}\left(\Delta_{o p t}^{*}\right)$ зависят от соотношения $\frac{\alpha}{\beta}$ :

$$
\Delta_{o p t}^{*}\left(\frac{\alpha}{\beta}\right)=\underset{\Delta^{*}[0, \alpha]}{\operatorname{Arg} \max } P_{\Pi \mathrm{P}}\left(\Delta^{*}, \frac{\alpha}{\beta}\right)=\underset{\Delta^{*} \in[0, \alpha]}{\operatorname{Arg} \min } P_{ת P}\left(\Delta^{*}, \frac{\alpha}{\beta}\right) .
$$

\section{Заключение}

В классических резервированных системах выбор оптимального значения порога $\Delta^{*}$ осуществляется из условия равенства вероятности ложного решения об отказе системы нулю

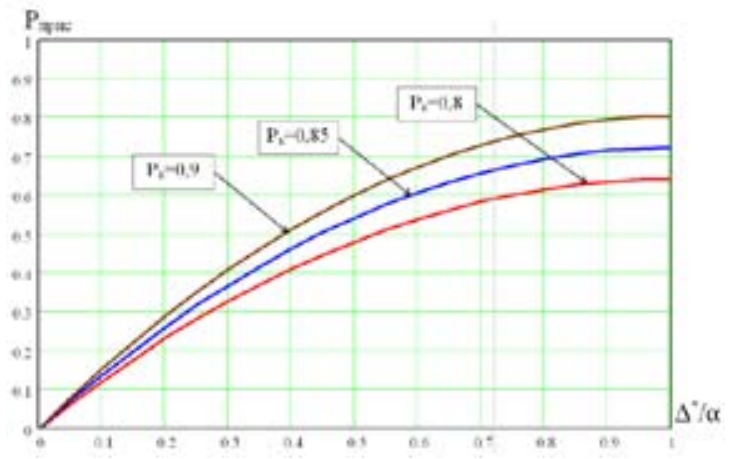

$a$

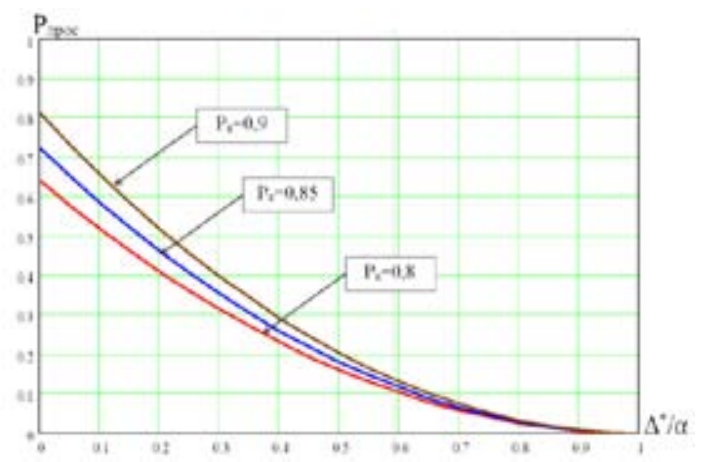

$\sigma$

Рис. 3. Зависимости вероятности правильного решения об исправности системы (а) и вероятности ложного решения об отказе системы (б) от отношения $\Delta^{*} / \alpha$ при фиксированных $P_{\text {к }}$ 


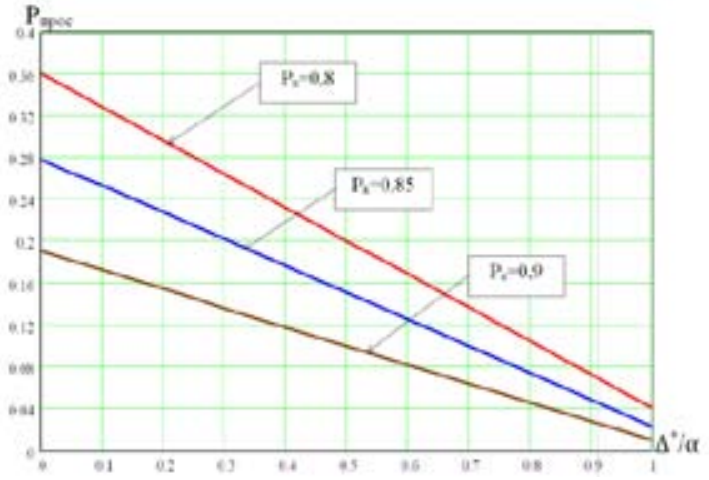

a

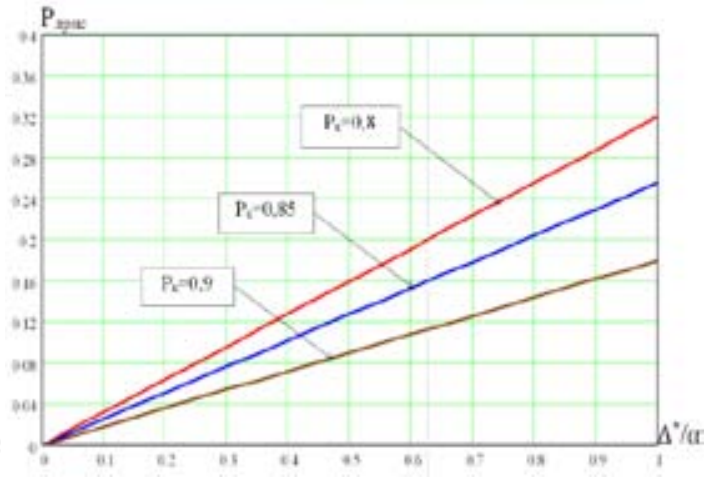

$\sigma$

Рис. 4. Зависимости вероятности правильного решения об отказе системы (а) и вероятности ложного решения об исправности системы (б) от отношения $\Delta^{*} / \alpha$ при фиксированных $P_{\text {к }}$

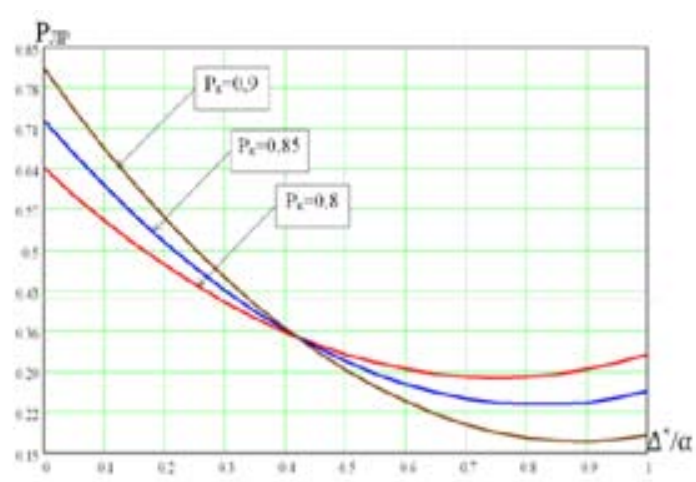

$a$

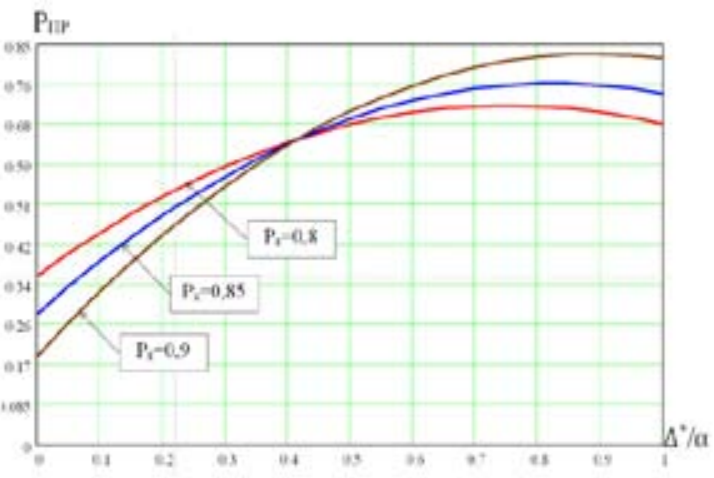

$\sigma$

Рис. 5. Зависимости вероятностей ложных решений (а) и правильных решений (б) от отношения $\Delta^{*} / \alpha$ при фиксированных $P_{\text {к }}$

$\left(\Delta^{*}=\alpha\right)$, так как в многоканальных резервированных системах (число каналов 3 и более) ложное решение об отказе системы приводит к исключению из дальнейшей работы исправного канала. В двухканальных резервированных системах, где факт обнаружения отказа является сигналом для подключения других устройств проверки, необходимо оптимизировать порог $\Delta^{*}$ таким образом, чтобы минимизировать вероятность принятия ложного решения об исправности системы при фиксированном максимально допустимом значении вероятности принятия ложного решения об отказе системы.

Выбор значения порога $\Delta^{*}$ в двухканальных резервированных системах следует с учетом отношения $\frac{\alpha}{\beta}$, что обеспечит максимум вероятности правильных решений и минимум вероятности ложных решений о состоянии резервированной системы. 


\section{Список литературы}

[1] Волобуев М.Ф., Замыслов М.А., Соколов И.В. Способ экономичного резервирования бортовых систем БЛА на основе нечеткой логики. Сб. статей II Всероссийской НПК «Академические Жуковские чтения» «Современные проблемы и перспективы развития комплексов авиационного оборудования». Воронеж: ВУНЦ ВВС «ВBA», 2015, 39-43 [Volobyev M.F., Zamislov M.A., Sokolov I.V. The method of economic onboard UAV reservation on the basis of indistinct logic. Art. Col. Of the second SPC «Zhukovsky academical readings» «Development of aviation equipment complexes modern problems and prospects». Voronezh, MESC AF «N.E. Zhukovsky and Y.A. Gagarin Air Force Academy», 2015, 39-43 (in Russian)].

[2] Шевцов Г.А., Шеремет Е.М. Логическое резервирование. Львов: Изд-во Львов. ун-та. 1973, 136 c. [Shevtsov A.G., Sheremet E.M. Logical reservation Lvov, Lvov university edition, 1973, 136 p. (in Russian)].

[3] Устройство управления резервированной с помощью мажоритарных элементов системой. Патент ФИПС РФ № 2451995 от 02.12.2011 г. [Reserved by majority elements system control device. Patent the Russian Federation № 2451995 since 02.12.2011 (in Russian)].

[4] Устройство обнаружения отказов в резервированной системе. Патент ФИПС РФ №2487389 от 20.06.2012 г. [Refusal detection device in the reserved system. Patent the Russian Federation №2487389 since 20.06.2012 (in Russian)].

[5] Устройство восстановления работоспособности системы резервированной с помощью мажоритарных элементов. Патент ФИПС РФ № 2493586 от 15.08 .2012 г. [Reserved by majority elements system workability restoration device. Patent the Russian Federation № 2493586 since 15.08.2012 (in Russian)].

[6] Венцель Е.С., Овчаров Л.А. Прикладные задачи теории вероятностей. М.: Радио и связь. 1983, 416 с. [Ventsel E.S., Ovcharov L.A. Probability theory applied tasks Moscow, Radio i sviaz, 1983, 416 p. (in Russian)]. 